\title{
A New Dedicated 256-Bit Hash Function: FORK-256
}

\author{
Deukjo Hong ${ }^{1}$, Donghoon Chang ${ }^{1}$, Jaechul Sung ${ }^{2}$, Sangjin Lee ${ }^{1}$, \\ Seokhie Hong ${ }^{1}$, Jaesang Lee ${ }^{1}$, Dukjae Moon ${ }^{3}$, and Sungtaek Chee ${ }^{3}$ \\ ${ }^{1}$ Center for Information Security Technologies(CIST), \\ Korea University, Seoul, Korea \\ \{hongdj, pointchang, sangjin, hsh, jslee\}@cist.korea.ac.kr \\ ${ }^{2}$ Department of Mathematics, University of Seoul, Seoul, Korea \\ jcsung@uos.ac.kr \\ ${ }^{3}$ National Security Research Institute \\ \{djmoon, chee\}@etri.re.kr
}

\begin{abstract}
This paper describes a new software-efficient 256-bit hash function, FORK-256. Recently proposed attacks on MD5 and SHA-1 motivate a new hash function design. It is designed not only to have higher security but also to be faster than SHA-256. The performance of the new hash function is at least 30\% better than that of SHA-256 in software. And it is secure against any known cryptographic attacks on hash functions.
\end{abstract}

Keywords: 256-bit Hash Function, FORK-256.

\section{Introduction}

For cryptographic hash function, the following properties are required:

- preimage resistance: it is computationally infeasible to find any input which hashes to any pre-specified output.

- second preimage resistance: it is computationally infeasible to find any second input which has the same output as any specified input.

- collision resistance: it is computationally infeasible to find a collision, i.e. two distinct inputs that hash to the same result.

For an ideal hash function with an $m$-bit output, finding a preimage or a second preimage requires about $2^{m}$ operations and the fastest way to find a collision is a birthday attack which needs approximately $2^{m / 2}$ operations.

Most dedicated hash functions which have iterative process use the MerkleDamgård construction [6]10] in order to hash inputs of arbitrary length. They work as follows. Let HASH be a hash function. The message $M$ is padded to a multiple of the block length and subsequently divided into $n$ blocks $M_{0}, \cdots$, $M_{n-1}$. Then HASH can be described as follows:

$$
C V_{0}=I V ; \quad C V_{i+1}=\operatorname{COMP}\left(C V_{i}, M_{i}\right), 0 \leq i \leq n-1 ; \quad \operatorname{HASH}(M)=C V_{n},
$$


where COMP is the compression function of $\mathrm{HASH}, C V_{i}$ 's are chaining variables, and $I V$ is a fixed initial value.

The most popular method of designing compression functions of dedicated hash functions is a serial successive iteration of a small step function, as like round functions of block ciphers. Many hash functions such as MD4 [12, MD5 [13], HAVAL [19, SHA-family [11, etc., follow that idea. Attacks on hash functions have been focused on vanishing the difference of intermediate values caused by the difference of messages. On the other hand, a hash function has been considered secure if it is computationally hard to vanish such difference in its compression function. Usually, the lower the probability of the differential characteristic is, the harder the attack is. Therefore a step function is regarded as a good candidate if it causes a good avalanche effect in the serial structure. A function which has a good diffusion property can not be so light in general. However, most step functions have been developed to be light for efficiency. This may be why MD4-type hash functions including SHA-1 are vulnerable to Wang et al.'s collision-finding attack [15/16/17/18].

RIPEMD-family 9] has somewhat different approach for designing a secure hash function. The attacker who tries to break members of RIPEMD-family should aim simultaneously at two ways where the message difference passes. This design strategy is still successful because so far there is not any effective attack on RIPEMD-family except the first proposal of RIPEMD. However, RIPEMD-family have heavier compression functions than hash functions with serial structure. For example, the first proposal of RIPEMD consists of two lines of MD4. Total number of steps is twice as many as that of MD4. Also, the number of steps of RIPEMD-160 is almost twice as many as that of SHA-0.

In this paper, we propose a new dedicated hash function FORK-256. According to the above observation, we determined the design goals (of compression function) as follows.

- It should have a 256-bit output because the security of $2^{128}$ operations is recommended for symmetric key cryptography as the computing power increases.

- Its structure should be resistant against known attacks including Wang et

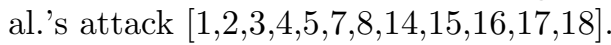

- The performance should be as competitive as that of SHA-256.

\section{Description of FORK-256}

In this section, we will describe FORK-256. These are basic notations used in FORK-256.

$\boxplus$ : addition $\bmod 2^{32}$

$\oplus:$ XOR (eXclusive OR)

$A^{\lll s}: s$-bit left rotation for a 32 -bit string $A$

$|A|_{512}$ : the number of 512-bit blocks in a string $A$ 


\subsection{Construction of FORK-256}

FORK-256 employs Merkle-Damgård construction with the compression function FORK256COMP $(\cdot, \cdot)$ and the padding method $\operatorname{PAD}(\cdot)$ as follows: For the initial value $C V_{0}=I V$ and the message $M$,

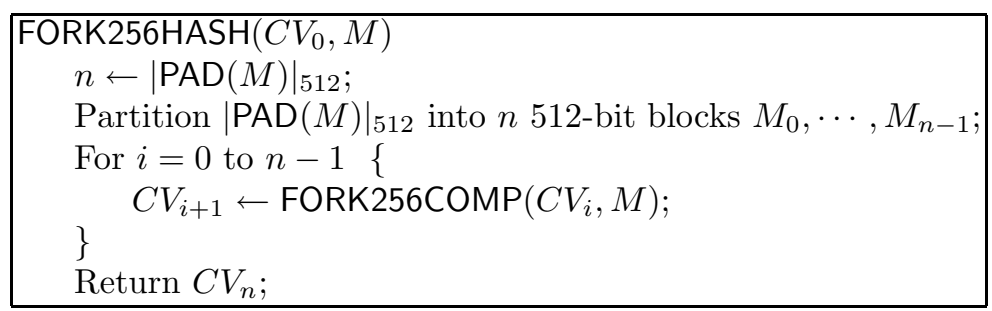

\subsection{Message Block Length and Padding}

The message block length of the compression function FORK256COMP is 512-bit. PAD pads a message by appending a single bit 1 next to the least significant bit of the message, followed by zero or more bit 0's until the length of the message is 448 modulo 512, and then appends to the message the 64-bit original message length modulo $2^{64}$.

\subsection{Structure of FORK-256 Compression Function}

Fig. 1 depicts the outline of the compression function FORK256COMP. The name 'FORK' was originated from the figure. FORK256COMP hashes a 512-bit string to a 256-bit string. It consists of four parallel branch functions, $\mathrm{BRANCH}_{1}$, BRANCH $_{2}$, BRANCH $_{3}$, and BRANCH B $_{4}$. Let $C V_{i}=\left(C V_{i}[0], C V_{i}[1], \cdots, C V_{i}[7]\right)$ where $C V_{i}[j]$ is a 32 -bit word. The initial value $C V_{0}$ is set as follows:

\begin{tabular}{ll}
\hline \hline$C V_{0}[0]=0 \times 6 \mathrm{a} 09 \mathrm{e} 667$ & $C V_{0}[1]=0 \times \mathrm{xbb} 67 \mathrm{ae} 85$ \\
$C V_{0}[2]=0 \times 3 \mathrm{c} 6 \mathrm{ef} 372$ & $C V_{0}[3]=0 \times 054 \mathrm{ff} 53 \mathrm{a}$ \\
$C V_{0}[4]=0 \times 510 \mathrm{e} 527 \mathrm{f}$ & $C V_{0}[5]=0 \times 9 \mathrm{~b} 05688 \mathrm{c}$ \\
$C V_{0}[6]=0 \times 1 \mathrm{f} 83 \mathrm{~d} 9 \mathrm{ab}$ & $C V_{0}[7]=0 \times 5 \mathrm{be} 0 \mathrm{~cd} 19$ \\
\hline \hline
\end{tabular}

Let us see the computing procedure of the $i$-th iteration of FORK256COMP. The message block $M_{i}$ is partitioned to 1632 -bit Words $\left(M_{i}[0], \cdots M_{i}[15]\right)$. Let $R_{j}^{(s)}=\left(R_{j}^{(s)}[0], \cdots, R_{j}^{(s)}[7]\right)$ for $1 \leq j \leq 4$ and $0 \leq s \leq 8$ where each $R_{j}^{(s)}[t]$ is a 32 -bit word for $0 \leq t \leq 7 . R_{j}^{(8)}$ is the output of $\mathrm{BRANCH}_{j}$ on the inputs $C V_{i}$ and $M_{i}$, for $1 \leq j \leq 4$ and computed as follows:

$$
R_{j}^{(8)}=\mathrm{BRANCH}_{j}\left(C V_{i}, M_{i}\right), \text { for } 1 \leq j \leq 4
$$

where $R_{j}^{(s)}$ 's are used in computation of $\mathrm{BRANCH}_{j}$ for $1 \leq j \leq 4$ and $0 \leq$ $s \leq 7$. Consequently, $C V_{i+1}=\left(C V_{i+1}[0], \cdots, C V_{i+1}[7]\right)$ is the output of the $i$-th iteration of FORK256COMP and computed as follows:

$$
C V_{i+1}[t]=C V_{i}[t] \boxplus\left(\left(R_{1}^{(8)}[t] \boxplus R_{2}^{(8)}[t]\right) \oplus\left(R_{3}^{(8)}[t] \boxplus R_{4}^{(8)}[t]\right)\right) \text {, for } 0 \leq t \leq 7 \text {. }
$$




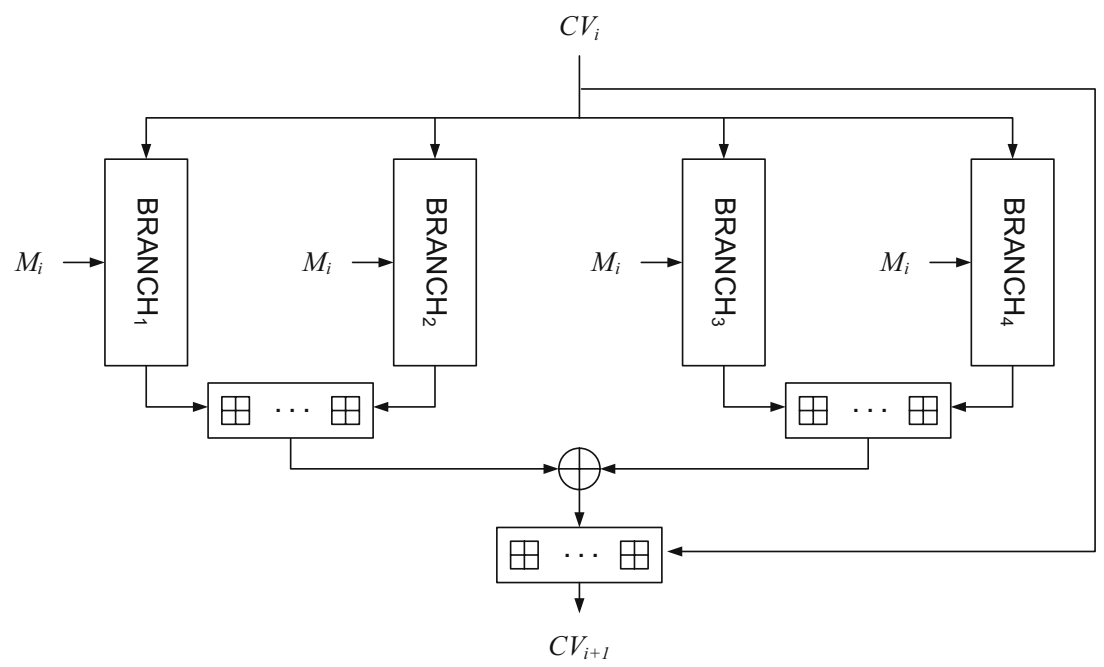

Fig. 1. Outline of the FORK-256 compression function, FORK256COMP

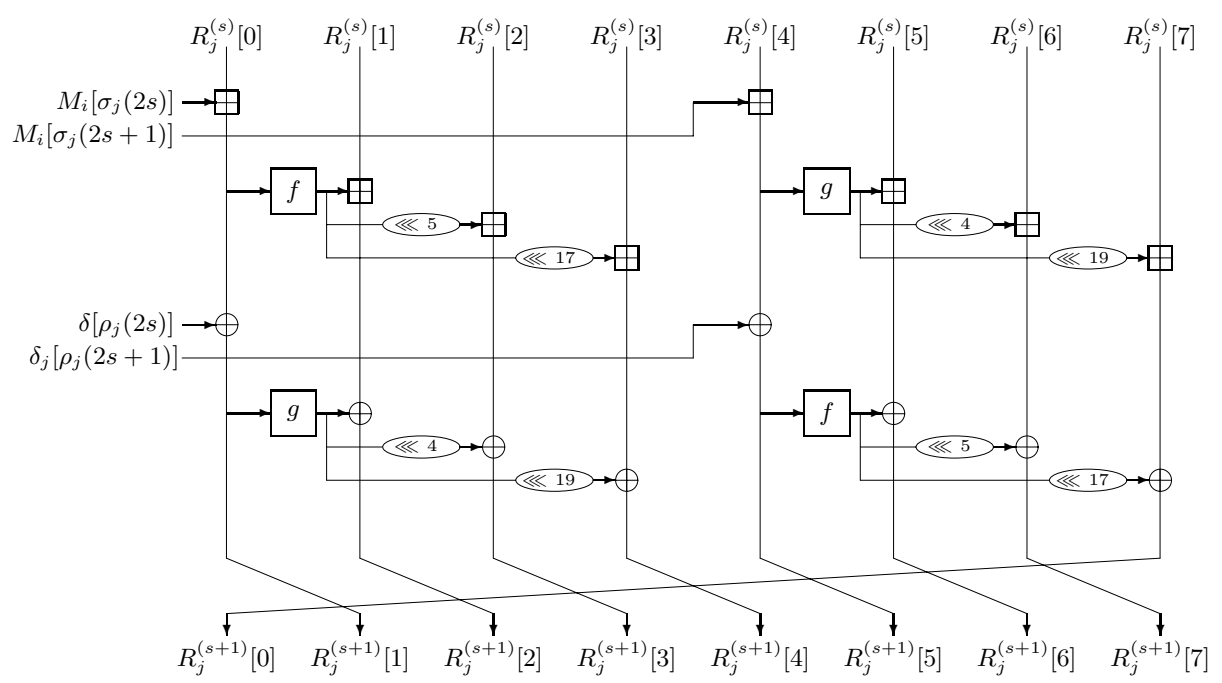

Fig. 2. Step function of FORK-256, STEP $(0 \leq s \leq 7,1 \leq j \leq 4)$

\subsection{Branch Function}

Each BRANCH Bro $_{j} 1 \leq j \leq 4$ is computed on the inputs $C V_{i}$ and $M_{i}$ as follows:

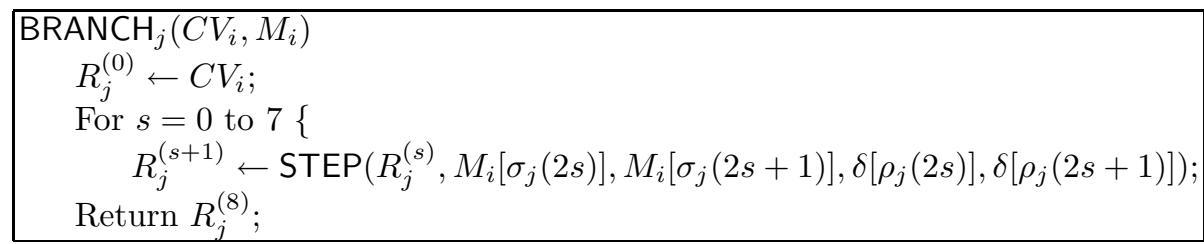


Message Word Ordering. Each $\mathrm{BRANCH}_{j}$ for $1 \leq j \leq 4$ uses the message words $M_{i}[0], \cdots, M_{i}[15]$ with different order $\sigma_{j}$.

Table 1. Message word ordering

\begin{tabular}{|c|c|c|c|c|c|c|c|c|c|c|c|c|c|c|c|c|}
\hline$s$ & 0 & 1 & 2 & 3 & 4 & 5 & 6 & 7 & 8 & 9 & 10 & 11 & 12 & 13 & 14 & 15 \\
\hline \hline$\sigma_{1}(s)$ & 0 & 1 & 2 & 3 & 4 & 5 & 6 & 7 & 8 & 9 & 10 & 11 & 12 & 13 & 14 & 15 \\
\hline$\sigma_{2}(s)$ & 14 & 15 & 11 & 9 & 8 & 10 & 3 & 4 & 2 & 13 & 0 & 5 & 6 & 7 & 12 & 1 \\
\hline$\sigma_{3}(s)$ & 7 & 6 & 10 & 14 & 13 & 2 & 9 & 12 & 11 & 4 & 15 & 8 & 5 & 0 & 1 & 3 \\
\hline$\sigma_{4}(s)$ & 5 & 12 & 1 & 8 & 15 & 0 & 13 & 11 & 3 & 10 & 9 & 2 & 7 & 14 & 4 & 6 \\
\hline
\end{tabular}

Constants. FORK256COMP totally uses sixteen constants:

\begin{tabular}{|c|c|c|c|}
\hline$\delta[0]$ & $=0 \mathrm{x} 428 \mathrm{a} 2 \mathrm{f} 98$ & 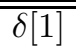 & $=0 \mathrm{x}$ \\
\hline$\delta[2]$ & $=0 \mathrm{xb} 5 \mathrm{c} 0 \mathrm{fbcf}$ & $\delta[3]$ & ba5 \\
\hline [4] & $25 b$ & $\delta[5]$ & $1 \mathrm{f}$ \\
\hline 6$]$ & $=0 \times 923$ & $\delta[7]$ & 5 ed5 \\
\hline$[8]$ & $=0 \mathrm{xd} 80$ & $\delta[9]$ & $5 \mathrm{~b} 01$ \\
\hline & $=0 \mathrm{x}$ & $\delta[11$ & c7dc3 \\
\hline$[10$ & $=0 \times 72 \mathrm{k}$ & $\delta[13$ & deb1fe \\
\hline 14 & $=0 \times 9 \mathrm{bdc} 06 \mathrm{a} 7$ & $\delta[15$ & $19 \mathrm{bf} 174$ \\
\hline
\end{tabular}

These constants are used in each BRANCH $\mathrm{BR}_{j}$ with different order $\rho_{j}$ for $1 \leq j \leq 4$.

Table 2. Constant ordering

\begin{tabular}{|c|c|c|c|c|c|c|c|c|c|c|c|c|c|c|c|c|}
\hline$s$ & 0 & 1 & 2 & 3 & 4 & 5 & 6 & 7 & 8 & 9 & 10 & 11 & 12 & 13 & 14 & 15 \\
\hline \hline$\rho_{1}(s)$ & 0 & 1 & 2 & 3 & 4 & 5 & 6 & 7 & 8 & 9 & 10 & 11 & 12 & 13 & 14 & 15 \\
\hline$\rho_{2}(s)$ & 15 & 14 & 13 & 12 & 11 & 10 & 9 & 8 & 7 & 6 & 5 & 4 & 3 & 2 & 1 & 0 \\
\hline$\rho_{3}(s)$ & 1 & 0 & 3 & 2 & 5 & 4 & 7 & 6 & 9 & 8 & 11 & 10 & 13 & 12 & 15 & 14 \\
\hline$\rho_{4}(s)$ & 14 & 15 & 12 & 13 & 10 & 11 & 8 & 9 & 6 & 7 & 4 & 5 & 2 & 3 & 0 & 1 \\
\hline
\end{tabular}

Step Function. In the $s$-th step of $\mathrm{BRANCH}_{j}$ for $1 \leq j \leq 4$ and $0 \leq s \leq 7$, STEP outputs $R_{j}^{(s+1)}$ on the inputs $R_{j}^{(s)}, M_{i}\left[\sigma_{j}(2 s)\right], M_{i}\left[\sigma_{j}(2 s+1)\right], \delta\left[\rho_{j}(2 s)\right]$, and $\delta\left[\rho_{j}(2 s+1)\right]$, and $R_{j}^{(s+1)}$ is computed as follows (See Fig. 2):

$$
\begin{aligned}
& R_{j}^{(s+1)}[0]= R_{j}^{(s)}[7] \boxplus g\left(R_{j}^{(s)}[4] \boxplus M_{i}\left[\sigma_{j}(2 s+1)\right]\right)^{\lll 21} \\
& \oplus f\left(R_{j}^{(s)}[4] \boxplus M_{i}\left[\sigma_{j}(2 s+1)\right] \boxplus \delta\left[\rho_{j}(2 s+1)\right]\right)^{\lll 17}, \\
& R_{j}^{(s+1)}[1]= R_{j}^{(s)}[0] \boxplus M_{i}\left[\sigma_{j}(2 s)\right] \boxplus \delta\left[\rho_{j}(2 s)\right], \\
& R_{j}^{(s+1)}[2]= R_{j}^{(s)}[1] \boxplus f\left(R_{j}^{(s)}[0] \boxplus M_{i}\left[\sigma_{j}(2 s)\right]\right) \\
& \oplus g\left(R_{j}^{(s)}[0] \boxplus M_{i}\left[\sigma_{j}(2 s)\right] \boxplus \delta\left[\rho_{j}(2 s)\right]\right),
\end{aligned}
$$




$$
\begin{gathered}
R_{j}^{(s+1)}[3]=R_{j}^{(s)}[2] \boxplus f\left(R_{j}^{(s)}[0] \boxplus M_{i}\left[\sigma_{j}(2 s)\right]\right)^{\lll 5} \\
\oplus g\left(R_{j}^{(s)}[0] \boxplus M_{i}\left[\sigma_{j}(2 s)\right] \boxplus \delta\left[\rho_{j}(2 s)\right]\right)^{\lll 9}, \\
R_{j}^{(s+1)}[4]=R_{j}^{(s)}[3] \boxplus f\left(R_{j}^{(s)}[0] \boxplus M_{i}\left[\sigma_{j}(2 s)\right]\right)^{\lll 17} \\
\oplus g\left(R_{j}^{(s)}[0] \boxplus M_{i}\left[\sigma_{j}(2 s)\right] \boxplus \delta\left[\rho_{j}(2 s)\right]\right)^{\lll 21}, \\
R_{j}^{(s+1)}[5]=R_{j}^{(s)}[4] \boxplus M_{i}\left[\sigma_{j}(2 s+1)\right] \boxplus \delta\left[\rho_{j}(2 s+1)\right], \\
R_{j}^{(s+1)}[6]=R_{j}^{(s)}[5] \boxplus g\left(R_{j}^{(s)}[4] \boxplus M_{i}\left[\sigma_{j}(2 s+1)\right]\right) \\
\oplus f\left(R_{j}^{(s)}[4] \boxplus M_{i}\left[\sigma_{j}(2 s+1)\right] \boxplus \delta\left[\rho_{j}(2 s+1)\right]\right), \\
R_{j}^{(s+1)}[7]=R_{j}^{(s)}[6] \boxplus g\left(R_{j}^{(s)}[4] \boxplus M_{i}\left[\sigma_{j}(2 s+1)\right]\right)^{\lll 9} \\
\oplus f\left(R_{j}^{(s)}[4] \boxplus M_{i}\left[\sigma_{j}(2 s+1)\right] \boxplus \delta\left[\rho_{j}(2 s+1)\right]\right)^{\lll 5},
\end{gathered}
$$

where $f$ and $g$ are nonlinear functions as follows:

$$
\begin{aligned}
& f(x)=x \boxplus\left(x^{\lll 7} \oplus x^{\lll 22}\right), \\
& g(x)=x \oplus\left(x^{\lll 13} \boxplus x^{\lll 27}\right) .
\end{aligned}
$$

\section{Design Strategy}

\subsection{Motivation for Our Proposal}

In 2004, Wang et al.'s attacks on MD4, MD5, HAVAL, and RIPEMD [15 16] and SHA-0/1 [17 18] brought a big impact on the field of symmetric key cryptography including hash function. However, RIPEMD-128/160 are the algorithms which are still secure against their attacks. No attacks on them are found so far.

They were designed to have two parallel lines, which is different from MD4, MD5 and SHA-family. This makes an attacker take into account two lines simultaneously. However, since each line needs almost same operation of MD5 and SHA algorithms, its efficiency was degenerated almost half of them. This motivates our design. We use four lines instead of two.

In order to overcome disadvantage of RIPEMD algorithms, we manage to reduce operations for step functions of each line. The message reordering of each branch is deliberately designed to be resistant against Wang et al.'s attack and differential attacks. The function $f$ and $g$ in each step are chosen to have good avalanche effects.

\subsection{Design Principle}

Structure. The compression function FORK256COMP consists of 4 Branches. In the security aspect, we can give the security against known attacks with the different message ordering in branches. For example, RIPEMD, which consists of 2 branches, was fully attacked by Wang et al. 15] because RIPEMD has the same message ordering in 2 branches. On the other hand, in case of RIPEMD$128 / 160$, there is no attack result because RIPEMD-128/160 have different message ordering in branches. In the implementation aspect, FORK-256 can 
be implemented efficiently because the message ordering is simpler than the message expansion such as that of SHA-256.

Constants. Each BRANCH${ }_{j}$ uses 16 different constants $\delta[t]$ for $0 \leq t \leq 15$. By using constants we pursue the goal to disturb the attacker who tries to find a good differential characteristic with a relatively high probability. So, we prefer the constants which represent the first 32 bits of the fractional parts of the cube roots of the first sixteen four prime numbers.

Nonlinear Functions. The nonlinear functions $f$ and $g$ of FORK256COMP output one word on the input of one word, and their outputs are XORed or added modulo $2^{32}$ to the multiple words in the chaining variables. Due to this property, $f$ and $g$ propagate the difference of a message word to the chaining variables.

Shift Rotations in Nonlinear Functions. If the addition is changed into the bitwise xor operation in $f$ and $g$, nonlinear functions are generalized as $x \oplus\left(x^{\lll s_{1}} \oplus x^{\lll s_{2}}\right)$. We consider all $465\left(=\left(\begin{array}{c}31 \\ 2\end{array}\right)\right)$ cases for $s_{1}$ and $s_{2}$ and want to define shift rotations satisfying the following 7 conditions. $\mathrm{HW}(w)$ denotes the Hamming Weight of a 32-bit word $w$. Let $x$ be an input of $f$ or $g$ and let $y$ be $f(x)$ or $g(x)$.

- The branch number of $f$ and $g$ is 4 .

- If $\mathrm{HW}(\mathrm{x})=2$, then $\mathrm{HW}(\mathrm{y}) \geq 4$.

- If $\mathrm{HW}(\mathrm{x})=3$, then $\mathrm{HW}(\mathrm{y}) \geq 3$.

- If $\mathrm{HW}(\mathrm{x})=4$, then $\mathrm{HW}(\mathrm{y}) \geq 4$.

- If $\mathrm{HW}(\mathrm{y})=1$, then $\mathrm{HW}(\mathrm{x}) \geq 17$.

- If $\mathrm{HW}(\mathrm{y})=2$, then $\mathrm{HW}(\mathrm{x}) \geq 14$.

- The interval of shift rotations are greater than or equal to 4 .

We determined the shift rotations such that $f$ and $g$ satisfy the above conditions.

Message Word Ordering. We adopt the message word ordering instead of the message word extension. If an attacker constructs an intended differential characteristics for one branch function, the ordering of message words will cause unintended differential patterns in the other branch functions. This is the core part of the security in the compression function. We considered the following four conditions in defining the message word ordering.

- Balance of upper (step 0 3) and lower (step 4 7) parts : each word should be applied twice to upper and lower parts, respectively.

- Balance of left and right parts : each word should be applied twice to left and right parts, respectively.

- Balance of sums of indices:

- Each word should be applied four times and indexed by $0 \sim 15$.

- Total sum of indices is 480 . Therefore, the average of sum of indices applied to each word is 30 . 
- We searched the ordering so that the sum of indices corresponding to each word is around $25 \sim 35$.

- Conditions which do not have same differential patterns in all branches:

- Specific differential pattern used at a branch may be applied to other branches.

- Therefore, except the case of giving a same difference to all words, we try to find an ordering such that there is no same differential patterns in all branches.

Shift Rotations and Rank. In the step function, 5 and 17, the values of shift rotation, are fixed. Then we search all the case and find candidate values (corresponding to 9 and 21) so that the rank of the linearly-changed step function is maximized. The maximum of the rank is 252 . Finally we select 9 and 21 among candidate values so that differences generated from the outputs of $f$ and $g$ functions do not overlap when a message word inserted at a step function has an one-bit difference.

\section{Security Analysis of FORK-256}

\subsection{Collision-Finding Attack}

We can analyze the collision-finding attacker's behavior in the aspect of message difference. Let $\Delta R_{j}^{(8)}$ be the output difference of $\mathrm{BRANCH}_{j}$ for $1 \leq j \leq 4$. Usually, the attacker expects the following event for finding collisions:

$$
\left(\Delta R_{1}^{(8)}[t] \boxplus \Delta R_{2}^{(8)}[t]\right) \oplus\left(\Delta R_{3}^{(8)}[t] \boxplus \Delta R_{4}^{(8)}[t]\right)=0, \quad \text { for } 0 \leq t \leq 7 .
$$

For this, he can take several strategies:

1. The attacker constructs a differential characteristic with high probability for a branch function, say BRANCH $\mathrm{B}_{1}$, and then expects that $\Delta R_{3}^{(8)}[t] \boxplus \Delta R_{4}^{(8)}[t] \boxminus$ $\Delta R_{2}^{(8)}[t]$ is equal to $\Delta R_{1}^{(8)}[t]$ for $0 \leq t \leq 7$.

2. The attacker constructs two distinct differential characteristics, and expects that $\Delta R_{1}^{(8)}=-\Delta R_{2}^{(8)}$ and $\Delta R_{3}^{(8)}=-\Delta R_{4}^{(8)}$ for $0 \leq t \leq 7$.

3 . The attacker inserts the message difference which yields same message difference pattern in four branches, and expects that same differential characteristic occurs simultaneously in four branches. Then the output difference of the compression function vanishes if the hamming weight of the output difference of each branch is small. This is because the final output is generated with using $\oplus$ and $\boxplus$ by turns.

Let us see the first strategy. If we assume that the outputs of each branch function is random, the probability of the event is almost close to $2^{-256}$. It is also difficult for the attacker to mount any attack following the second strategy because he should find such differential pattern of the message words. 
Third strategy is relatively easy for the attacker to perform. For example, if he inserts the same difference to all the message words, then the same message difference pattern occurs in every branches. However, the message word reordering was designed so that the third strategy is satisfied only if the attacker inserts the same difference to all the message words. Under the assumption that every step is independent, we can compute the upper bound of the probability that such kind of differential characteristic occurs, which frustrates the attacker.

\subsection{Attacks Using Inner Collision Patterns}

When the attacker inserts the differences to the message words, the event that the difference of the intermediate value becomes zero often occurs. It is called inner collision. We call a differential characteristic which causes an inner collision with a probability, inner collision pattern.

Note that an inner collision is not a real collision, but the notion of inner collision pattern is important in cryptanalysis of hash function because it can be repeatedly used to yield a real collision with a high probability. The main idea of attacks on SHA-0 and SHA-1 is also the repetition of an inner collision pattern. So, in hash functions with a serial structure it is related to the resistance against collision-finding attack how many time an inner collision can be repeated.

Let us focus on only one branch function. We omit the subscript index in the variables. We can construct 5 -step inner collision pattern easily. Let $\Delta R^{(s)}[t]$ be the difference of $R^{(s)}[t]$, and let $\Delta M[\sigma(i)]$ be the difference of $M[\sigma(i)]$. Table 3 and 4 show two among 5-step inner collision patterns in one branch function, which we found. They holds with the probability $2^{-40}$, respectively.

If we apply these patterns to $\mathrm{BRANCH}_{1}$, the output difference $\Delta R_{1}^{(8)}$ will be zero with the probability $2^{-40}$. As mentioned in the previous subsection, however, it is hard to use the pattern for the attack on FORK-256 because the following events seldom occurs: either that the computation of the output differences of

Table 3. Case 1. 5-step inner collision pattern in a branch: The numbers in the entries of the table denotes the bits in which the difference is 1

\begin{tabular}{|c||l|l|l|l|l|}
\hline $\mathrm{s}$ & 0 & 1 & 2 & 3 & 4 \\
\hline \hline$\Delta M[\sigma(2 s)]$ & 31 & & & & \\
\hline$\Delta M[\sigma(2 s+1)]$ & & $1,6,15,16,20,23$ & $3,4,8,11,21,26$ & $6,12,21,26$ & 31 \\
\hline$\Delta R^{(s)}[0]$ & & & & & \\
\hline$\Delta R^{(s)}[1]$ & & 31 & & & \\
\hline$\Delta R^{(s)}[2]$ & & $6,12,21,26$ & 31 & & \\
\hline$\Delta R^{(s)}[3]$ & & $3,4,8,11,21,26$ & $6,12,21,26$ & 31 & \\
\hline$\Delta R^{(s)}[4]$ & & $1,6,15,16$ & $3,4,8,11,20,23$ & $6,12,21,26,21,26$ & 31 \\
\hline$\Delta R^{(s)}[5]$ & & & & & \\
\hline$\Delta R^{(s)}[6]$ & & & & & \\
\hline$\Delta R^{(s)}[7]$ & & & & & 1 \\
\hline \hline Prob. & $2^{-10}$ & $2^{-16}$ & $2^{-10}$ & $2^{-4}$ & \\
\hline
\end{tabular}


Table 4. Case 2. 5-step inner collision pattern in a branch: The numbers in the entries of the table denotes the bits in which the difference is 1

\begin{tabular}{|c||l|l|l|l|l|}
\hline $\mathrm{s}$ & 0 & 1 & 2 & 3 & 4 \\
\hline \hline$\Delta M[\sigma(2 s)]$ & & $1,6,15,16,20,23$ & $3,4,8,11,21,26$ & $6,12,21,26$ & 31 \\
\hline$\Delta M[\sigma(2 s+1)]$ & 31 & & & & \\
\hline$\Delta R^{(s)}[0]$ & & $1,6,15,16,20,23$ & $3,4,8,11,21,26$ & $6,12,21,26$ & 31 \\
\hline$\Delta R^{(s)}[1]$ & & & & & \\
\hline$\Delta R^{(s)}[2]$ & & & & & \\
\hline$\Delta R^{(s)}[3]$ & & & & & \\
\hline$\Delta R^{(s)}[4]$ & & & & & \\
\hline$\Delta R^{(s)}[5]$ & & 31 & & & \\
\hline$\Delta R^{(s)}[6]$ & & $6,12,21,26$ & 31 & & \\
\hline$\Delta R^{(s)}[7]$ & & $3,4,8,11,21,26$ & $6,12,21,26$ & 31 & \\
\hline \hline Prob. & $2^{-10}$ & $2^{-16}$ & $2^{-10}$ & $2^{-4}$ & 1 \\
\hline
\end{tabular}

the other branches is zero or that the other branches have the same differential pattern in the message words as BRANCH $\mathrm{Br}_{1}$.

\section{$5 \quad$ Efficiency and Performance}

In this section we compare the total number of operations and the performance of FORK-256 and SHA-256. The total number of operations is compared in the Table 5 .

Table 5. Number of operations used in FORK-256 and SHA-256

\begin{tabular}{|c||c|c|}
\hline operation & FORK-256 & SHA-256 \\
\hline \hline addition $(+)$ & 472 & 600 \\
\hline bitwise operation $(\oplus, \wedge, \vee)$ & 328 & 1024 \\
\hline shift $(\ll, \gg)$ & - & 96 \\
\hline shift rotation $(\lll, \ggg)$ & 512 & 576 \\
\hline
\end{tabular}

Table 6. Performance of FORK-256 and SHA-256

\begin{tabular}{|c||c|c|c|c|}
\hline \multirow{2}{*}{ environment } & \multicolumn{2}{|c|}{ FORK-256 } & \multicolumn{2}{c|}{ SHA-256 } \\
\cline { 2 - 5 } & Mbps & Cycle/Byte & Mbps & Cycle/Byte \\
\hline Pen3/WinXP/VC & 192.010 & 31.413 & 132.469 & 44.581 \\
\hline Pen4/WinXP/VC & 521.111 & 28.755 & 318.721 & 46.372 \\
\hline
\end{tabular}

Table 6] shows the performance of FORK-256 and SHA-256 in the two environments. The environments are denoted by $C P U / O S / C o m p i l e r$ and the following notations are used in description of environments for simplicity. 
Pen3 : Pentium III, $801 \mathrm{MHz}$

Pen4 : Pentium IV, $2.0 \mathrm{GHz}$

WinXP : Microsoft Windows XP Professional ver 2002

VC : Microsoft Visual C++ Ver 6.0

\section{Summary}

In this paper we have proposed a new dedicated 256-bit hash function FORK256. The main features are the followings;

- The structure of the compression function consists of 4 parallel branch functions.

- Nonlinear functions $f$ and $g$ are quite different from the boolean functions which have been used in existing hash functions, and updates multiple words.

- The ordering of the message words is simple but well organized such that it is very difficult for any attacker to find good inner collision patterns.

According to our security analysis, FORK-256 looks resistant against existing attacks including Wang et al.'s attack, but we encourage the readers to give any further security analysis. Finally, our performance test shows that the performance of FORK-256 is faster than that of SHA-256, and we expect that the difference between the performance of FORK-256 and SHA-256 would increase after optimization.

\section{Acknowledgement}

This research was supported by the MIC(Ministry of Information and Communication), Korea, under the ITRC(Information Technology Research Center) support program supervised by the IITA(Institute of Information Technology Assessment).

\section{References}

1. E. Biham and R. Chen, "Near-Collisions of SHA-0," Advances in Cryptology CRYPTO 2004, LNCS 3152, Springer-Verlag, pp. 290-305, 2004.

2. E. Biham, R. Chen, A. Joux, P. Carribault, C. Lemuet and W. Jalby, "Collisions of SHA-0 and Reduced SHA-1," Advances in Cryptology - EUROCRYPT 2005, LNCS 3494, Springer-Verlag, pp. 36-57, 2005.

3. B. den Boer and A. Bosselaers, "An Attack on the Last Two Rounds of MD4," Advances in Cryptology - CRYPTO'91, LNCS 576, Springer-Verlag, pp. 194-203, 1992.

4. B. den Boer and A. Bosselaers, "Collisions for the Compression Function of MD5," Advances in Cryptology - CRYPTO'93, LNCS 765, Springer-Verlag, pp. 293-304, 1994.

5. F. Chabaud and A. Joux, "Differential Collisions in SHA-0," Advances in Cryptology - CRYPTO'98, LNCS 1462, Springer-Verlag, pp. 56-71, 1998. 
6. I. Damgård, "A Design Priciple for Hash Functions," Advances in Cryptology CRYPTO'89, LNCS 435, Springer-Verlag, pp. 416-427, 1989.

7. H. Dobbertin, "RIPEMD with Two-Round Compress Function is Not CollisionFree," Journal of Cryptology 10:1, pp. 51-70, 1997.

8. H. Dobbertin, "Cryptanalysis of MD4," Journal of Cryptology 11:4, pp. 253-271, 1998.

9. H. Dobbertin, A. Bosselaers and B. Preneel, "RIPEMD-160, a strengthened version of RIPEMD," FSE'96, LNCS 1039, Springer-Verlag, pp. 71-82, 1996.

10. R. C. Merkle, "One way hash functions and DES," Advances in Cryptology CRYPTO'89, LNCS 435, Springer-Verlag, pages 428-446, 1989.

11. NIST/NSA, "FIPS 180-2: Secure Hash Standard (SHS)", August 2002 (change notice: February 2004).

12. R. L. Rivest, "The MD4 Message Digest Algorithm," Advances in Cryptology CRYPTO'90, LNCS 537, Springer-Verlag, pp. 303-311, 1991.

13. R. L. Rivest, "The MD5 Message-Digest Algorithm," IETF Request for Comments, RFC 1321, April 1992.

14. B. Van Rompay, A. Biryukov, B. Preneel and J. Vandewalle, "Cryptanalysis of 3pass HAVAL," Advances in Cryptology - ASIACRYPT 2003, LNCS 2894, SpringerVerlag, pp. 228-245, 2003.

15. X. Wang, X. Lai, D. Feng, H. Chen and X. Yu, "Cryptanalysis of the Hash Functions MD4 and RIPEMD," Advances in Cryptology - EUROCRYPT 2005, LNCS 3494, Springer-Verlag, pp. 1-18, 2005.

16. X. Wang and H. Yu, "How to Break MD5 and Other Hash Functions," Advances in Cryptology - EUROCRYPT 2005, LNCS 3494, Springer-Verlag, pp. 19-35, 2005.

17. X. Wang, H. Yu and Y. L. Yin, "Efficient Collision Search Attacks on SHA-0," Advances in Cryptology - CRYPTO 2005, LNCS 3621, Springer-Verlag, pp. 1-16, 2005.

18. X. Wang, Y. L. Yin and H. Yu, "Finding Collisions in the Full SHA-1," $A d$ vances in Cryptology - CRYPTO 2005, LNCS 3621, Springer-Verlag, pp. 17-36, 2005.

19. Y. Zheng, J. Pieprzyk and J. Seberry, "HAVAL - A One-Way Hashing Algorithm with Variable Length of Output," Advances in Cryptology - AUSCRYPT'92, LNCS 718, Springer-Verlag, pp. 83-104, 1993.

\section{A Source Code}

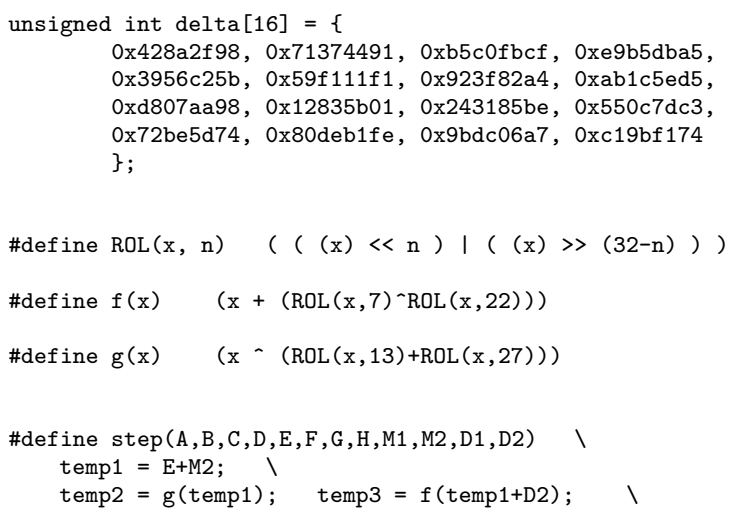




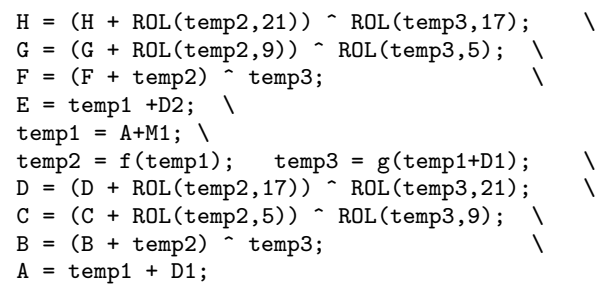

static void FORK256_Compression_Function(unsigned int *CV, unsigned int $*$ M) \{

unsigned long R1[8] ,R2 [8] ,R3[8] ,R4 [8];

unsigned long temp1, temp2, temp3;

$\mathrm{R} 1[0]=\mathrm{R} 2[0]=\mathrm{R} 3[0]=\mathrm{R} 4[0]=\mathrm{CV}[0] ;$
$\mathrm{R} 1[1]=\mathrm{R} 2[1]=\mathrm{R} 3[1]=\mathrm{R} 4[1]=\mathrm{CV}[1] ;$
$\mathrm{R} 1[2]=\mathrm{R} 2[2]=\mathrm{R} 3[2]=\mathrm{R} 4[2]=\mathrm{CV}[2] ;$
$\mathrm{R} 1[3]=\mathrm{R} 2[3]=\mathrm{R} 3[3]=\mathrm{R} 4[3]=\mathrm{CV}[3] ;$
$\mathrm{R} 1[4]=\mathrm{R} 2[4]=\mathrm{R} 3[4]=\mathrm{R} 4[4]=\mathrm{CV}[4] ;$
$\mathrm{R} 1[5]=\mathrm{R} 2[5]=\mathrm{R} 3[5]=\mathrm{R} 4[5]=\mathrm{CV}[5] ;$
$\mathrm{R} 1[6]=\mathrm{R} 2[6]=\mathrm{R} 3[6]=\mathrm{R} 4[6]=\mathrm{CV}[6] ;$
$\mathrm{R} 1[7]=\mathrm{R} 2[7]=\mathrm{R} 3[7]=\mathrm{R} 4[7]=\mathrm{CV}[7] ;$

// BRANCH1 (CV,M)

step(R1 [0] ,R1 [1] ,R1 [2] ,R1 [3] ,R1 [4] ,R1 [5] , R1 [6] ,R1 [7] , M [0] , M [1] , delta [0] , delta [1] ); $\operatorname{step}(\mathrm{R} 1[7], R 1[0], R 1[1], R 1[2], R 1[3], R 1[4], R 1[5], R 1[6], M[2], M[3], d e l t a[2], d e l t a[3])$; step(R1 [6] ,R1 [7] ,R1 [0] ,R1 [1] ,R1 [2] ,R1 [3] ,R1 [4] ,R1 [5] , M [4] , M [5] , delta [4] , delta [5] ); step (R1 [5] , R1 [6] , R1 [7] , R1 [0] , R1 [1] , R1 [2] , R1 [3] , R1 [4] , M [6] , M [7] , delta [6] ,delta [7] ); step (R1 [4] ,R1 [5] ,R1 [6] ,R1 [7] ,R1 [0] ,R1 [1] ,R1 [2] ,R1 [3] , M [8] , M [9] , delta [8] , delta [9] ); step (R1 [3] , R1 [4] , R1 [5] , R1 [6] , R1 [7] , R1 [0] , R1 [1] , R1 [2] , M [10] , M [11] , delta [10] , delta [11]) ; step (R1 [2] , R1 [3] , R1 [4] , R1 [5] , R1 [6] , R1 [7] , R1 [0] , R1 [1] , M[12] , M[13] , delta [12], delta [13]); step(R1 [1] ,R1 [2] ,R1 [3] , R1 [4] ,R1 [5] ,R1 [6] , R1 [7] , R1 [0] ,M [14] ,M [15] , delta [14] , delta [15] ) ;

\section{// BRANCH2 (CV, M)}

step (R2 [0] , R2 [1] , R2 [2] , R2 [3] , R2 [4] , R2 [5] , R2 [6] , R2 [7] , M [14] , M [15] , delta [15] , delta [14] ) ; step (R2 [7] , R2 [0] , R2 [1] , R2 [2] , R2 [3] , R2 [4] , R2 [5] , R2 [6] , M [11] , M [9] , delta [13] , delta [12] ) ; step (R2 [6] , R2 [7] , R2 [0] , R2 [1] , R2 [2] , R2 [3] , R2 [4] , R2 [5] ,M [8] , M[10], delta [11], delta[10]); step (R2 [5] ,R2 [6] ,R2 [7] ,R2 [0] ,R2 [1] ,R2 [2] ,R2 [3] ,R2 [4] , M [3] , M [4] , delta [9] , delta [8] ); step (R2 [4] , R2 [5] , R2 [6] , R2 [7] , R2 [0] , R2 [1] , R2 [2] , R2 [3] , M [2] , M [13] , delta [7] , delta [6] ) ; step (R2 [3] ,R2 [4] ,R2 [5] ,R2 [6] ,R2 [7] , R2 [0] ,R2 [1] ,R2 [2] , M [0] , M [5] , delta [5] , delta [4] ); step (R2 [2] , R2 [3] , R2 [4] , R2 [5] , R2 [6] , R2 [7] , R2 [0] , R2 [1] , M [6] , M [7] , delta [3] , delta [2] ); step (R2 [1] , R2 [2] , R2 [3] , R2 [4] , R2 [5] , R2 [6] , R2 [7] , R2 [0] ,M [12] , M[1] , delta [1] , delta [0] );

\section{// BRANCH3 (CV,M)}

step (R3 [0] , R3 [1] , R3 [2] , R3 [3] , R3 [4] , R3 [5] , R3 [6] , R3 [7] , M [7] , M [6] , delta [1] , delta [0] ) ; step (R3 [7] ,R3 [0] , R3 [1] , R3 [2] , R3 [3] , R3 [4] , R3 [5], R3 [6] , M [10] , M [14] , delta [3] , delta [2] ) ; step (R3 [6] , R3 [7] , R3 [0] , R3 [1] , R3 [2] , R3 [3] , R3 [4] , R3 [5] , M [13] , M [2] , delta [5] , delta [4] ); step (R3 [5] , R3 [6] , R3 [7] , R3 [0] , R3 [1] , R3 [2] , R3 [3] , R3 [4] , M [9] , M [12], delta [7] , delta [6] ) ; step (R3 [4] , R3 [5] , R3 [6] , R3 [7] , R3 [0] , R3 [1] , R3 [2] , R3 [3] , M [11] , M [4] , delta [9] , delta [8] ); step (R3 [3] , R3 [4] , R3 [5] , R3 [6] , R3 [7] , R3 [0] , R3 [1] , R3 [2] , M[15] , M [8] , delta [11] , delta [10] ); step (R3 [2] , R3 [3] , R3 [4] , R3 [5] , R3 [6] , R3 [7] , R3 [0] , R3 [1] , M [5] , M [0] , delta [13] , delta [12] ); $\operatorname{step}(R 3[1], R 3[2], R 3[3], R 3[4], R 3[5], R 3[6], R 3[7], R 3[0], M[1], M[3], \operatorname{delta}[15], \operatorname{delta}[14]$ ) ;

\section{// BRANCH4 (CV, M)}

step (R4 [0] , R4 [1] , R4 [2] , R4 [3] , R4 [4] , R4 [5] , R4 [6] , R4 [7] , M [5] , M [12] , delta [14] , delta [15] ) ; step (R4 [7] , R4 [0] , R4 [1] , R4 [2] , R4 [3] , R4 [4] , R4 [5] , R4 [6] , M [1] , M [8] , delta [12] , delta [13] ) ; step (R4 [6] , R4 [7] , R4 [0] , R4 [1] ,R4 [2] , R4 [3] , R4 [4] , R4 [5], M[15], M [0], delta [10], delta [11]); step (R4 [5] , R4 [6] , R4 [7] , R4 [0] , R4 [1] , R4 [2] , R4 [3] , R4 [4] , M [13] , M [11] , delta [8], delta [9] ) ; step (R4 [4] , R4 [5] , R4 [6] , R4 [7] , R4 [0] , R4 [1] , R4 [2] , R4 [3] , M [3] , M [10], delta [6] , delta [7] ) ; step (R4 [3] , R4 [4] , R4 [5] , R4 [6] , R4 [7] , R4 [0] , R4 [1] , R4 [2] , M [9] , M [2] , delta [4] , delta [5] ); step (R4 [2] , R4 [3] , R4 [4] , R4 [5] , R4 [6] , R4 [7] , R4 [0] , R4 [1] , M [7] , M [14], delta [2] , delta [3] ) ; step (R4 [1] , R4 [2] , R4 [3] , R4 [4] , R4 [5] , R4 [6] , R4 [7] , R4 [0] , M [4], M [6], delta [0] , delta [1]); 


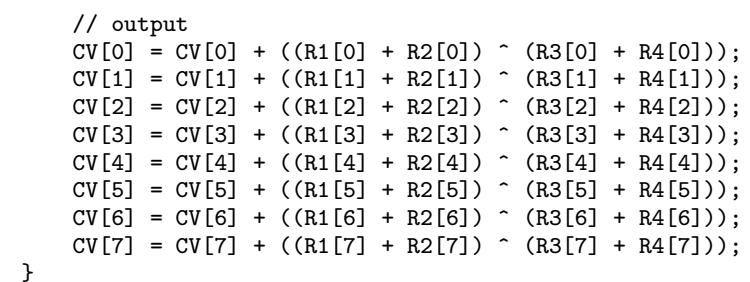

\section{B Test Vector}

Message $M$ (1 block)

4105ba8c d8423ce8 ac484680 07ee1d40 bc18d07a 89fc027c 5ee37091 cd1824f0 878de230 dbbaf0fc da7e4408 c6c05bc0 330650207367 cfc5 f4aa5c78 e1cbc780

\section{Output of Compression Function $C V_{1}$}

ebcc5b3d d3715534 a6a7a68a e6022b02 49c676ed 639a34b0 b8d978c2 cfdf1a2b

\section{Intermediate Values}

$\mathrm{BRANCH}_{1}$

$R_{1}^{(0)}=6 a 09$ e667 bb67ae85 3c6ef372 a54ff53a 510e527f 9b05688c 1f83d9ab 5be0cd19

$R_{1}^{(1)}=574 \mathrm{faabb}$ ed99d08b 55559509 ca832197 cc3e5d3d 9a87d3f8 a53a7eff e5b76844

$R_{1}^{(2)}=15 \mathrm{~b} 6 \mathrm{~cd} 3 \mathrm{~d}$ b958ed0a bc5ec9da 0685ff8e eecd75a9 bde25622 $730387 \mathrm{f0} 8 \mathrm{~cd} 537 \mathrm{f} 4$

$R_{1}^{(3)}=\mathrm{b} 37 \mathrm{a} 2 \mathrm{f} 3 \mathrm{c}$ 0b266012 421e26a6 c78f6e0b $1 \mathrm{~cd} 85800 \mathrm{~d} 2 \mathrm{ba} 8 \mathrm{a} 16 \quad 7449 \mathrm{f} 6 \mathrm{c} 0$ 0f8c7a01

$R_{1}^{(4)}=31$ be4596 a49d2271 6ee14e1a e33ff108 11f5f01a 950cdbc5 5dcd1a2a 32aa199f

$R_{1}^{(5)}=62 \mathrm{fd} 9 \mathrm{~d} 8 \mathrm{~b} 9153 \mathrm{~d} 25 \mathrm{e} 4 \mathrm{a} 23586 \mathrm{e} 9 \mathrm{~b} 599483 \mathrm{cf} 29 \mathrm{e} 3 \mathrm{af} 00343 \mathrm{c} 17 \mathrm{f} 33 \mathrm{f} 23 \mathrm{cb} 9 \mathrm{c} 903 \mathrm{e} 62$

$R_{1}^{(6)}=\mathrm{d} 36228 \mathrm{e} 461 \mathrm{ad} 6751$ fe55bb69 $94720 \mathrm{~b} 3 \mathrm{c} 8 \mathrm{a} 810 \mathrm{aa} 7$ eaf6bd32 $737155 \mathrm{e} 2 \mathrm{~b} 96 \mathrm{a} 93 \mathrm{e} 9$

$R_{1}^{(7)}=7 \mathrm{a} 779 \mathrm{e} 327926 \mathrm{~d} 678$ 3aec6bdd 0e208057 c349f555 7ec78c6a 91ebeb68 1fc96600

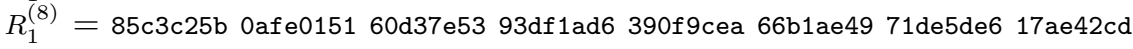

\section{$\mathrm{BRANCH}_{2}$}

$R_{2}^{(0)}=6 \mathrm{a} 09 \mathrm{e} 667$ bb67ae85 3c6ef372 a54ff53a 510e527f 9b05688c 1f83d9ab 5be0cd19 $R_{2}^{(1)}=09 \mathrm{a} 80 \mathrm{c} 1 \mathrm{a} 20503453 \mathrm{~b} 7 \mathrm{ce} 65 \mathrm{dc} 686 \mathrm{c} 5844$ 8f7b750a ceb620a6 e84808f4 13a2716f $R_{2}^{(2)}=$ e21fd29c 514719d8 47c2c8b0 116c12a7 42ddee6f ddf4c37a 3b2884ee 1b6552ca $R_{2}^{(3)}=608 \mathrm{f} 85 \mathrm{bc}$ beba328f da492019 ce8cc5ac e939ee3d 418db835 0d4088c0 a4515753 $R_{2}^{(4)}=9 \mathrm{~d} 8199357 \mathrm{~b} 00 \mathrm{fdfd}$ d9947c55 0dfccfd7 817088d7 7d5a694f 8da6b62e 3b63944f $R_{2}^{(5)}=\mathrm{f} 22 \mathrm{fa} 55 \mathrm{e}$ f4e63e8a $2516289 \mathrm{f} 77 \mathrm{~d} 9 \mathrm{~b} 888 \mathrm{dc500533} 8717 \mathrm{db} 40 \quad 6158 \mathrm{e} 3 \mathrm{e} 7 \quad 0 \mathrm{e} 922286$ $R_{2}^{(6)}=13 \mathrm{ca} 89 \mathrm{c} 4$ 8d2671db afbc022b 9580fdfe 356e2f63 9fa2ca0a d2199dee 455937e5 $R_{2}^{(7)}=$ b8d0fc67 5c63d5fa d2b45236 fad40792 759b52ab b8475022 1cf6c001 6a0cf5f2 $R_{2}^{(8)}=08283$ ecb 5d0e9118 da92c996 9316c47c $261673589067 \mathrm{bf} 2 \mathrm{~b} 33 \mathrm{a} 76294$ a2c36255

$\mathrm{BRANCH}_{3}$ $R_{3}^{(0)}=6 a 09 e 667$ bb67ae85 3c6ef372 a54ff53a 510e527f 9b05688c 1f83d9ab 5be0cd19 $R_{3}^{(1)}=46 \mathrm{f} 81 \mathrm{ba} 6$ a8594fe8 f0348c97 749c040f 8e6801dc f27bf2a8 275472bf 0866407e $R_{3}^{(2)}=56 \mathrm{a} 9 \mathrm{eac} 1$ 0b2c3b53 0e98c271 ec010b6c $448475 \mathrm{~b} 538 \mathrm{~d} 35 \mathrm{a} 23$ 455b10c5 4c819e3b 
$R_{3}^{(3)}=38 \mathrm{~cd} 29 \mathrm{dc} 2402 \mathrm{cc} 7748018 \mathrm{a} 70$ 26a5dcf2 3da527e9 2a237e90 2f4dc6a8 33bd5b6f $R_{3}^{(4)}=$ a28f637c bfa479ad $68059737374 \mathrm{a} 7 \mathrm{e} 75$ b5e5b8c6 02eafaad 15799680 ae2d5da0 $R_{3}^{(5)}=64607852$ 7bd31a3d a54f54b2 4013d658 1fbcbc0a 4a0633d8 $972027 \mathrm{f} 7$ 40a519ed $R_{3}^{(6)}=$ b27cf46d 9b38bd95 fb3978fd d52a18c8 1cdbd155 cb7c23f8 d3ce2cdd 5e6705b2 $R_{3}^{(7)}=317 \mathrm{ce} 148$ bd57a8e7 d3b60337 f0dd8789 1a925421 d09fe955 c626a195 8d38ed5d $R_{3}^{(8)}=72$ ec7187 cb5b0fa4 59b04096 55b45924 d54c20ad be5c7808 ec104b46 08d57f3d

$\mathrm{BRANCH}_{4}$ $R_{4}^{(0)}=6 \mathrm{a} 09 \mathrm{e} 667$ bb67ae85 3c6ef372 a54ff53a 510e527f 9b05688c 1f83d9ab 5be0cd19 $R_{4}^{(1)}=$ ce371d88 8fe1ef8a f4e6891a dd47fbec $8655 \mathrm{e} 36945 \mathrm{~b} 09413$ 8d2e660f 968ed897 $R_{4}^{(2)}=015 \mathrm{a} 57 \mathrm{e} 3$ 1937b7e4 d82e18fe 374895df 3e1357d6 8ec27797 81e87c75 627d168a $R_{4}^{(3)}=$ f2619dce $0757 \mathrm{a} 521$ b3dc348f a91771d4 00a58535 d4259025 37fc2a18 c5a9d37a $R_{4}^{(4)}=$ dc4ebcd3 3dd1182b acb226cd 3ed1c4a9 f6191a1b d9e93bf6 $62752 \mathrm{a} 33$ d29d946e $R_{4}^{(5)}=$ ad2c36d3 767c5cb7 $8 \mathrm{~d} 977401$ ebd447de a0e6e49b 7bb3bcf8 d7b3eadc 71c2d2a 4 $R_{4}^{(6)}=$ b871dbb2 c23dea2a aebfcf21 6de34a20 41d677c5 a7203d0c 14c00db6 d5b6d5ce $R_{4}^{(7)}=\mathrm{a} 6072510$ 3b4afc71 e74b9db3 5120200b b1167426 2036afe2 ddcd1ac5 096735bb $R_{4}^{(8)}=99420469$ a4aa2522 f7aeb45b 10939176 d252137f 81312948 50c01427 c0ba68f3 